\title{
Entornos de Aprendizaje Autoorganizados para el desarrollo de competencias básicas en Ciencias Naturales
}

Self-Organized Learning Environments for the development of basic skills in Natural Sciences

\author{
María Angélica \\ Cancio Nisperuza \\ Magister en Educación \\ Institución Educativa El Siglo \\ Colombia \\ magyi282@hotmail.com
}

\author{
Geiner De Jesús \\ Montes Miranda \\ Magister en Educación \\ Institución Educativa Guateque \\ Colombia \\ geinermontes@hotmail.com
}

\author{
Mary Luz \\ Doria Rojas \\ Magister en Educación \\ Universidad de Córdoba \\ Colombia \\ mldoria@correo.unicordoba.edu.co
}

\section{Resumen}

La educación en Colombia presenta diferentes problemáticas en lo referente a calidad, cobertura e infraestructura escolar; específicamente, en el área de las ciencias naturales se evidencian métodos poco creativos y obsoletos que no estimulan el aprendizaje. Motivados por contribuir al cambio, se diseñó una investigación cuyo objetivo fue analizar el efecto de la implementación de la metodología Self Organized Learning Environment (SOLE) como modelo de trabajo para la indagación, la explicación y la aplicación de lo aprendido en ciencias en los estudiantes del grado octavo "A" de la Institución Educativa El Siglo del municipio de Ciénega de Oro, departamento de Córdoba (Colombia). Con un diseño metodológico de corte cuantitativo y de tipo cuasiexperimental seleccionando un grupo control y un grupo experimental. La investigación pretendió contribuir a la enseñanza de las ciencias naturales en contexto, poniendo en práctica procesos cognitivos, procedimentales y personales. Se evidenciaron diferencias estadísticamente significativas entre el grupo control y el grupo experimental, en las clases tradicionales de ciencias naturales hubo poca participación mientras que con SOLE los estudiantes fueron gestores de su

Palabras claves:

SOLE, competencias básicas, ciencias naturales, aprendizaje. aprendizaje, trabajaron en equipo y se apoyaron para lograr el objetivo; además, se mejoró el nivel de desempeño, pasando de mínimo a satisfactorio; mientras que el grupo control inició y se mantuvo en insuficiente.

Recepción: Diciembre 09 de 2019 | Aceptación: Marzo 12 de 2020 | Publicación: Junio 30 de 2020 


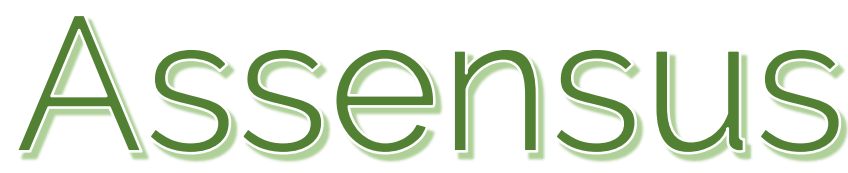

Revista de Investigación educativa y pedagógica

\section{Abstract}

Keywords:

SOLE, basic skills, natural sciences, learning.
Education in Colombia presents different problems in terms of quality, coverage and school infrastructure; specifically, in the area of natural sciences, uncreative and obsolete methods that do not stimulate learning are evident. Motivated by this change, an investigation was designed to analyze the effect generated by the implementation of the SOLE methodology (self-organized learning environments) on the level of development of basic natural science competencies in eighth grade "A" students at the El Siglo Educational Institution in the municipality of Ciénega de Oro, department of Córdoba (Colombia). With a methodological design of quantitative cut and quasi-experimental type where a control group and an experimental group were selected. This research has sought to contribute to the teaching of Natural Sciences in context, putting into practice cognitive, procedural and personal processes. It was evidenced that there was a statistically significant difference between the control group and the experimental group, in the traditional classes of natural sciences there was little participation while with SOLE the students were managers of their learning, worked in team and were supported to achieve the objective; in addition, the level of performance was improved, going from minimum to satisfactory; while the control group began and remained insufficient.

Received: December 09, 2020 | Accepted: March 12, 2020 | Published: June 30, 2020 


\section{Introducción}

La educación en Colombia presenta diferentes problemáticas en lo referente a calidad, cobertura e infraestructura escolar que derivan de la administración educativa y la continuidad de un modelo de educación tradicional, que ha intentado ajustarse a los cambios sociales, pero sin cambiar las funciones y roles específicos de las formas de enseñanza y aprendizaje. Específicamente en las ciencias naturales este problema es consecuencia de la prolongación de métodos poco creativos que no estimulan el aprendizaje y que, en muchos casos, implementan una pedagogía tradicional que a veces se caracteriza por el regaño, la humillación y el castigo por parte de los docentes hacía los estudiantes (Camacho, 2018, p.45).

Así mismo, Lopata y Schittner (2014, p.56) sostienen que al interior de las instituciones educativas colombianas se evidencian serias limitaciones en el desarrollo de estrategias y prácticas didácticas apropiadas que integren el uso de herramientas tecnológicas ajustadas a las necesidades de un ambiente globalizado. Al respecto, Camacho (2018, p.67) expresa que se observan estrategias rudimentarias por parte de docentes y alumnos en las cuales no se optimizan los recursos disponibles; algunas veces por resistencias inherentes de los sujetos ante las propuestas de cambio, y otras, por falta de herramientas e ideas que les permitan desarrollar prácticas educativas innovadoras y significativas en el contexto escolar.

Sumado a lo anterior, Arteaga, Armada y Del Sol (2016, p.169), consideran que la enseñanza de las ciencias requiere de profundas transformaciones pero no al estilo adaptativo, sino al estilo innovador, de manera que el profesor deje de ser un transmisor de conocimientos y tome conciencia de su función como creador de posibilidades para que el alumno produzca y construya el conocimiento; es así como la enseñanza de las ciencias tiene el deber de preparar al hombre para la vida y esto se logra desarrollando métodos y estrategias de aprendizaje que permitan la búsqueda del conocimiento a partir de situaciones tomadas del entorno y donde se pueda apreciar la aplicación de la ciencia en la vida.

Por otra parte, si bien en la Institución Educativa El Siglo del municipio de Ciénaga de Oro se han realizado procesos de mejoramiento en el plan de estudio, mallas curriculares, planes de aula y en general, en el Proyecto Educativo Institucional (PEI) con el fin de adaptarse a las necesidades actuales y lograr una transformación de las practicas educativas, los resultados no se han acercado a los esperados, puesto que los estudiantes siguen mostrando dificultades en las actividades que se realizan en el aula de clases.

Aunque se han mostrado avances en lo referente a la configuración del PEI, esto no ha sido suficiente; en la práctica, aspectos como la misión, visión, perfil del estudiante, desarrollo de contenidos e incluso, en los preparadores de algunos docentes, lo plasmado en el papel no corresponde a la realidad de las aulas; tal como lo exponen Murillo, Hernández y Martínez (2016, p.123), se ha observado que la enseñanza se centra en la reproducción de contenidos, algunos docentes reducen la intención pedagógica de sus acciones y la interacción con los alumnos, lo único que solicitan de ellos son las respuestas correctas de las actividades 
realizadas, no es habitual que el docente plantee reflexiones al grupo y las tareas sólo tienen una forma de realizarse correctamente. En este sentido, se ha observado que los estudiantes de la Institución Educativa El Siglo están recibiendo clases a través de una metodología tradicional, lo que supondría que algunos docentes no se están preocupando por introducir nuevas formas de enseñanza que sean atractivas para los educandos de tal manera que se favorezca su aprendizaje. Por tal razón, desde el estudio se planteó determinar la relación entre la implementación de la estrategia SOLE y el nivel de desarrollo de las competencias, uso comprensivo del conocimiento, explicar e indagar en las ciencias naturales en los estudiantes del grado octavo "A" de la Institución Educativa El Siglo del municipio de Ciénega de Oro, departamento de Córdoba (Colombia).

De acuerdo con esto, este artículo derivado de la investigación mencionada, se estructura de la siguiente manera: una introducción, donde se explica la problemática estudiada, los objetivos, los fundamentos teóricos, seguidamente se profundiza sobre los referentes metodológicos que orientaron el desarrollo de la investigación y, posteriormente, se muestran los resultados obtenidos en el estudio investigativo. Finalmente, la discusión y las principales conclusiones a las que se llegaron con todo el proceso realizado.

\section{Referentes teóricos}

La investigación se fundamentó desde conceptualizaciones como la enseñanza, la cual es considerada como una actividad socio comunicativa y cognitiva que dinamiza los aprendizajes significativos en ambientes ricos y complejos (aula, aula virtual, aula global o fuera del aula), síncrona o asíncronamente (Sarmiento, 2004, p.98). Por su parte, Cousinet, (2014, p.3) refiere que enseñar es presentar a los alumnos y hacerles adquirir, en vista de una conservación problemática conocimientos nuevos; es ayudarlos a tratar de conocer mejor lo que ellos ya conocen, y por consiguiente lo que ellos desean conocer mejor.

Por otro lado, son múltiples las concepciones que existen sobre el aprendizaje; sin embargo, para motivos de la presente investigación y de acuerdo a Zapata (2015, p.74) se definió como un proceso o conjunto de procesos a través del cual o de los cuales, se adquieren o se modifican ideas, habilidades, destrezas, conductas o valores, como resultado del estudio, la experiencia, la instrucción, el razonamiento o la observación. Según Sarmiento (2004, p.56) el aprendizaje permite darle un significado y valor al conocimiento, de forma que pueda ser representado y transmitido a otros individuos remota y atemporalmente mediante códigos complejos dotados de estructura (lenguaje escrito, códigos digitales, entre otros); es decir, lo que unos aprenden puede ser utilizado por otros en otro lugar o en otro tiempo.

De igual forma, la enseñanza y el aprendizaje de las Ciencias Naturales constituyen una preocupación creciente en el ámbito educativo, en particular, por la necesidad de pensar su funcionalidad en la sociedad actual. Es de gran importancia que los alumnos logren explicaciones verdaderas acerca de los fenómenos naturales y de los conocimientos sobre los 
seres que habitamos el planeta, ello, para que resuelvan problemas con eficiencia. Por tal motivo, es tarea del docente mantener en sus alumnos la curiosidad por conocer y aprender, experimentar, preguntar y observar, todo esto lo puede lograr actualizándose constantemente para conocer métodos y estrategias que puedan emplear en el ambiente escolar y poder lograr un aprendizaje significativo (González, 2007, p.120).

Así mismo, los entornos de aprendizaje autoorganizados (o SOLE por sus siglas en inglés: Self Organized Learning Environment) invitan a los estudiantes a trabajar de forma colaborativa para generar un aprendizaje significativo, siendo parte activa, constructiva y resolviendo un problema, dando como producto la autoorganización del conocimiento. Según Ortega (2014, p.31), estos entornos consisten en un modelo educativo basado en aprendizaje colaborativo, donde los estudiantes, de forma conjunta, buscan, aclaran y construyen su propio conocimiento mediante la colaboración mutua en el aula de clases.

\section{Metodología}

La investigación asumió un enfoque cuantitativo donde es posible estudiar la asociación o relación entre las variables cuantificadas para hacer inferencias en la población de la cual procede la muestra (Pita \& Pértigas; 2002); la cual en algunos casos se obtiene sin aleatorización de los sujetos que conforman los grupos. En este caso, se trabajó con un diseño cuasi experimental con grupos intactos, en el cual se tuvo un grupo control (sin intervención) y uno experimental al que se le aplicó la metodología de ambientes de aprendizaje autoorganizados para conocer si esta tenía efectos en los niveles de desarrollo de las competencias básicas de las ciencias naturales que son evaluadas mediante pruebas externas (uso comprensivo del conocimiento, indagar y explicar).

El proyecto se desarrolló en la Institución Educativa EI Siglo, ubicada en el corregimiento El Siglo, aproximadamente a $12 \mathrm{~km}$ del casco urbano del municipio de Ciénaga de Oro, departamento de Córdoba. Es una institución de carácter público rural, con dos jornadas (mañana y tarde), cuenta con cuatro sedes anexas (EI Salado, Venado, El Bobo y Egipto) y una sede principal (El Siglo). Tiene una población de 1001 estudiantes, de los cuales 483 pertenecen a primaria y 518 a secundaria, con un promedio de 30 estudiantes por aula. El grado octavo de la institución educativa se distribuye en cuatro grupos (A, B, C y D) con un total de 120 estudiantes de acuerdo al reporte del Sistema Integrado de Matricula. La muestra fue seleccionada de forma no probabilística y correspondió a 60 estudiantes pertenecientes al grado octavo que se encontraban legalmente matriculados en el año 2019, distribuidos en los grupos A y B. Estos jóvenes se encuentran en edades entre los 13 y 16 años, están en una etapa de gran actividad motriz y donde sus habilidades creativas se encuentran en un nivel óptimo para ser aprovechadas. A pesar de las limitaciones económicas y las carencias de su entorno, la mayoría demuestra interés por aprender y superarse.

Para escoger la muestra, se tuvo en cuenta que ambos grupos presentaran condiciones similares en cuanto a rendimiento académico, número de estudiantes, situaciones 
disciplinarias y características socioeconómicas, escogiendo al grado octavo A $\left(8^{\circ} \mathrm{A}\right)$ como el grupo experimental y al grado octavo $\mathrm{B}\left(8^{\circ} \mathrm{B}\right)$ como el grupo control. En esta investigación el 46,6 \% (28 estudiantes) fueron hombres, mientras que el restante 53,3 \% (32 estudiantes) correspondieron a mujeres.

\section{Procedimiento}

Se diseñó una encuesta estructurada con la escala de Likert con el fin de conocer la percepción de los estudiantes sobre los métodos de enseñanza que se implementaban en la Institución Educativa El Siglo y una prueba escrita tipo examen Saber con la cual se determinó el nivel de desempeño de las competencias básicas de ciencias naturales. A los instrumentos se les evaluó su confiabilidad con el Alfa de Cronbach, obteniendo valores de 97,8 \% para la encuesta y $86,2 \%$ en el test tipo prueba Saber, la cual a su vez fue validada sometiéndola al juicio de tres expertos (todos con el grado de maestría) quienes dieron un puntaje del 85,16 $\%$ y una valoración de excelente.

Una vez conocida la percepción de los estudiantes sobre los métodos de enseñanza que los docentes de ciencias naturales aplicaban en el aula y determinado el nivel de desarrollo de las competencias básicas (uso comprensivo del conocimiento, explicar e indagar) con la aplicación del pretest, se procedió a la elaboración de las sesiones de clases en consistencia con el modelo SOLE para su aplicación en el grupo experimental (Octavo A) durante dos meses, mientras que el grupo control (Octavo B) siguió con sus clases de forma tradicional. En la planificación de las sesiones se utilizó la aplicación StartSOLE, esta es una app que permitió descargar y compartir información sobre como introducir los SOLE en el aula, obtener las grandes preguntas que inicialmente se implementaron con los estudiantes y diseñar las sesiones de trabajo para posteriormente compartirlas con otras personas vinculadas a la red educativa de SOLE. Las sesiones SOLE fueron planeadas de acuerdo a las recomendaciones del manual de implementación de SOLE Colombia (2016) y tuvieron la siguiente secuencia: introducción, elaboración de grandes preguntas, búsqueda y retroalimentación.

Finalmente se aplicó el postest, el cual consistió en tres sesiones, cada una de 25 minutos y cuyo tiempo fue tomado de las horas de clase asignadas a la docente. Por sesión se resolvieron en el aula de clases 10 peguntas del test prueba Saber, seleccionada de forma aleatoria y con variaciones en las opciones de respuesta de forma que no se influenciaran los resultados por la memorización de las preguntas por parte de los estudiantes. El postest se aplicó en ambos grupos (experimental y control) finalizada la intervención con la estrategia SOLE.

Los resultados de la encuesta y del test tipo prueba Saber, se tabularon utilizando estadística descriptiva para identificar tendencias y rangos de las diferentes respuestas de los estudiantes. Además, para determinar el estado de desarrollo de cada competencia básica de ciencias naturales, tanto en el pretest como en el postest de los grupos experimental y control se tuvieron en cuenta los niveles establecidos por el ICFES (2018). Se agruparon las preguntas del test por competencias y se calificaron tomando como nota máxima el valor de 100; de igual forma, a cada estudiante se le otorgó una calificación general en cada una de 
las pruebas realizadas y se consolidaron los datos para cada grupo. De acuerdo con los resultados de cada estudiante, estos se situaron en uno de los niveles de desempeño previamente establecidos (I, II, III O IV). Posteriormente, se analizaron los resultados discriminándolos por competencias y también se determinó el nivel general de desempeño de los grupos experimental y control tomando como punto de referencia el promedio de los puntajes obtenidos por los estudiantes pertenecientes a cada grupo.

Se evaluó la normalidad de los datos mediante el test de Shapiro-Wilk $(\alpha<0,05)$. La comprobación de las hipótesis y la determinación de posibles diferencias significativas entre el nivel de desarrollo de las competencias básicas de ciencias naturales de los grupos experimental y control se realizó con la prueba U de Mann Whitney (Addinosoft, 2014), esta prueba se ajusta a un modelo no paramétrico y es empleada cuando existen dos muestras las cuales son independientes. La prueba también fue realizada en cada competencia básica de ciencias naturales.

Finalmente, se aplicó la prueba de Wilcoxon con el fin de determinar posibles diferencias significativas intragrupo; es decir, en este punto se comparó consigo mismo el grupo control y el grupo experimental en el pretest y el postest.

\section{Resultados}

A partir de los datos obtenidos con la encuesta y con la recolección de los testimonios de los estudiantes se pudo determinar que en la Institución Educativa El Siglo del municipio de Ciénaga de Oro predominan las metodologías de enseñanza tradicional, se estableció que la mayoría de los estudiantes consideraron que a pesar que los docentes tienen un buen manejo de las temáticas del grado, casi siempre se sentían desmotivados en las clases debido a que en su mayoría la temática no se muestra de forma interesante y tampoco resulta llamativa. Asimismo, expresaron que falta brindarles una mayor participación durante las clases, dado que, en muchas ocasiones solo el docente tiene la palabra y la razón, casi siempre las clases se basan en transcribir información proporcionada por el docente del texto guía o desarrollar talleres escritos, favoreciendo la monotonía y la distracción y, dejando de lado, la experimentación, la búsqueda de información y el descubrimiento de las soluciones a problemas de su contexto.

Por otro lado, en cuanto a las didácticas innovadoras tales como el uso de internet, herramientas tecnológicas, trabajos grupales, salidas a campo y prácticas de laboratorio, la mayoría de los encuestados consideraron que se emplean escasamente y solo para abordar algunos temas. De igual forma, con la encuesta quedó en evidencia que, para los estudiantes los aprendizajes que se obtienen mediante clases tradicionales son limitados y en ocasiones no se profundiza la temática por no contar con los recursos y lo visto en clase no tiene un significado o aplicabilidad. 
El análisis de frecuencia permitió establecer que, a nivel general, de las 1260 respuestas obtenidas de los estudiantes (60 estudiantes x 21 ítems) el 42,5 \% correspondió al nivel de A veces, mientras que el 26,7 \% de las respuestas fueron Nunca. Por su parte, el 22,0 \% de las respuestas fueron Casi siempre y el restante $8,9 \%$ fueron Siempre; lo anterior ratifica que la mayoría de los estudiantes consideran que las clases de ciencias naturales se enfocan a métodos tradicionales que no favorecen o maximizan los aprendizajes y donde el docente proporciona la información y los estudiantes son meros receptores de esta sin que exista una verdadera retroalimentación entre los actores.

Al analizar las diferentes estrategias pedagógicas empleadas por los docentes en clases de ciencias naturales se pudo determinar que el $35 \%$ usa las consultas en casa, mientras que el $16,7 \%$ emplea talleres, seguido de salidas al tablero con 15,0 \% y trabajos escritos con el $11,7 \%$. Estas son estrategias de corte tradicional y se han dejado de lado acciones como el uso de elementos virtuales, las prácticas de laboratorio, el aprendizaje por problemas, las dinámicas y la implementación de clases más motivantes.

En cuanto a los diferentes instrumentos y ejercicios de evaluación de los aprendizajes que se usan en el área de ciencias naturales, el 41,7 \% de estudiantes del grado octavo sostuvo que son evaluados principalmente mediante evaluaciones escritas y el 18,3\% afirmó que es mediante evaluaciones orales. Por su parte, el 15,0 \% y el 13,3 \% expresaron que se les evalúa con salidas al tablero y talleres respectivamente y, solo el $3 \%$ de los estudiantes consideraron que se emplean autoevaluaciones para verificar su aprendizaje. Esta última es una estrategia que se inició a implementar en todas las áreas de formación de la Institución Educativa El Siglo a partir del segundo semestre del año 2019 como parte de la estrategia de mejoramiento institucional de la gestión académica y con el fin de dinamizar los procesos evaluativos de la institución. Ninguno de los estudiantes consideró que en las clases de ciencias se le evalúa con rubricas, listas de chequeo o que se tienen en cuenta sus habilidades o destrezas.

Para los recursos didácticos empleados en las clases de ciencias naturales estableció que para la mayor parte de estudiantes emplea el tablero (58,3 \%, 35 estudiantes) y el texto guía (21,7 \%, 13 estudiantes), generalmente manejado solo por el docente y se entregan copias de partes específicas a los estudiantes para que las desarrollen o transcriban al cuaderno sin que responda a un verdadero objetivo de aprendizaje. Por otro lado, solo el 8,3 \% (5 estudiantes) consideró que en clases de ciencias se emplean videos o animaciones referentes a la temática y solo un estudiante (1,7 \%) expresó que en clases se recurre al uso de computadores y del aula de laboratorio.

En el pretest, al realizar el contraste de hipótesis intergrupos con la prueba $U$ de Mann Whitney, se pudo establecer que no existieron diferencias significativas entre el grupo control y el grupo experimental en el pretest (U: 3480,5; p-valor: 0,0991), lo que indica que las condiciones iniciales de ambos grupos eran similares.

Al comparar los resultados obtenidos en el pretest se pudo determinar que a nivel general el grupo control tuvo una calificación de 38,2 situándolo en un nivel insuficiente, mientras que el grupo experimental tuvo un valor medio de 41,9; lo que indica que los estudiantes se 
situaron en el nivel de desempeño mínimo; sin embargo, como se mencionó estos resultados no fueron estadísticamente diferentes.

Al comparar los resultados por competencias básicas entre los grupos del pretest se observa que el mayor valor se logró en la competencia indagación por parte en el grupo experimental (49 puntos; nivel mínimo) y el menor valor se obtuvo en la competencia uso comprensivo del conocimiento por parte del grupo control (35 puntos; nivel insuficiente). De igual forma, se evidenció que en las preguntas del instrumento relacionadas con las competencias explicación y uso comprensivo del conocimiento fue donde los estudiantes presentaron mayor dificultad para su realización. En dichas competencias, tanto el grupo control como el experimental se situaron en un nivel insuficiente, lo que demuestra que los estudiantes muy posiblemente no lograron superar las preguntas de menor complejidad que se realizaron en la prueba.

Por su parte, en el postest los resultados del grupo control y el grupo experimental mediante la prueba de Mann-Whitney se determinó que existieron diferencias estadísticamente significativas entre los resultados de la prueba del grupo control vs el grupo experimental (U: 1598,0; p-valor < 0,0001), evidenciándose que para el grupo experimental hubo un avance significativo mientras que el grupo control no registró un progreso en su desempeño.

Luego de la aplicación de la estrategia SOLE en el grupo experimental y la continuación de las clases de forma tradicional en el grupo control, se evidenció una marcada diferencia entre los resultados obtenidos en el postest. A nivel general para el grupo control se obtuvo un promedio de 36,3; lo que lo sitúa en un nivel insuficiente; dicho valor fue inferior al obtenido en el pretest $(38,2)$, aunque estuvo dentro del mismo nivel de desempeño (Insuficiente). Por su parte, el grupo experimental obtuvo un promedio general de 58,4; este valor es mayor al obtenido inicialmente en el pretest $(41,9)$, asimismo, se pasó de un nivel de desempeño mínimo en el pretest a un nivel satisfactorio en el postest. Dichos resultados dejan en evidencia que la aplicación de SOLE como estrategia para el mejoramiento del desempeño estudiantil en el área de ciencias naturales fue beneficiosa.

Al aplicar la prueba de Mann Whitney para cada competencia básica de ciencias naturales se observa que en todos los casos existe diferencia significativa entre los resultados del grupo control y el grupo experimental ( $p$-valor $<0,05$ ); por tanto, para cada una de las hipótesis especificas planteadas se puede concluir que se debe rechazar la hipótesis nula y aceptar la hipótesis alternativa, dejando claro que existe diferencia estadística significativa en los resultados de cada competencia básica de ciencias naturales.

Por otro lado, al comparar los resultados obtenidos para cada competencia básica de ciencias naturales de los grupos evaluados, se determinó que el grupo experimental superó ampliamente en todas las competencias al grupo control situándose todos los resultados en el nivel satisfactorio (entre 56 y 70 puntos); en la competencia indagación se logró el mayor valor con 61 puntos, seguido de la competencia uso compresivo del conocimiento con 58 puntos y de explicación de fenómenos con 56,3 puntos de 100 posibles. En contraste a esto, 
el grupo control no tuvo un progreso en cuanto al desarrollo de competencias, situándose todas en el nivel insuficiente (entre cero y 40 puntos).

Al igual que en el pretest, en el postest se evidenció que la mayor dificultad del grupo control se situó en la competencia uso comprensivo del conocimiento (35 puntos en el pretest y 30 en el postest), en las competencias indagación y explicación los resultados disminuyeron, lo que puede ser indicio que las clases tradicionales generaron un efecto negativo en la adquisición de conocimiento por parte de los estudiantes, olvidándose conceptos y no existiendo una apropiación adecuada de estos.

La comparación intragrupo mediante la prueba de Wilcoxon, permitió determinar que no existieron diferencias significativas entre los resultados obtenidos en el pretest y el postest del grupo control (V: 1513,0; p-valor: 0,4958), mientras que en el grupo experimental, al comparar los resultados del pretest y el postest, los resultados fueron estadísticamente diferentes (V: 519,5; p-valor< 0,0001), motivo por el cual, en este grupo se rechazó la hipótesis nula y se aceptó la hipótesis alternativa. Lo anterior también se estableció en los niveles de desempeño, en los que el grupo control se mantuvo en un nivel insuficiente, mientras que el grupo experimental, luego de la intervención con la estrategia SOLE, pasó de un nivel de desempeño mínimo a un nivel satisfactorio.

\section{Discusión}

Mediante la encuesta de percepción estudiantil y en conversaciones con los docentes se pudo determinar que en la Institución Educativa El Siglo del municipio de Ciénaga de Oro predominan las metodologías de enseñanza de corte tradicional; se evidenció que a pesar que los docentes se esmeran por planear sus clases, por desarrollar las temáticas completamente y aunque los contenidos se encuentran acorde con lo establecido por el MEN, no siempre son contextualizados o transversalizados, muchas veces se desarrollan de forma mecánica, dando mayor importancia a la cantidad sobre la calidad, esto a pesar que los lineamientos curriculares del área de ciencias naturales apuntan a un enfoque más significativo e integral. Lo anterior y de acuerdo a Fernández, Tuset, Pérez y Leyva (2009, p.145) muchas veces es debido a la resistencia al cambio por parte de los docentes, resistencia que tiene su origen en las concepciones de algunos maestros sobre sobre la enseñanza y el aprendizaje; muchas veces, basados en ideas erróneas porque se rigen por un realismo ingenuo, según el cual la simple exposición al contenido o al objeto del aprendizaje garantizará el resultado, concebido como una reproducción de la información presentada. Este tipo de lógica hace que las concepciones constructivistas del aprendizaje y la enseñanza resulten profundamente contraintuitivas y, por tanto, difíciles de asumir por los maestros.

Según lo observado en la presente investigación y de acuerdo a Daza y Moreno (2010, p.39) muchos maestros parten de lo establecido en los planes de área, estándares básicos de competencias y presaberes para planear sus clases; sin embargo, centran su trabajo y el desempeño de los estudiantes en lo académico más que en la experiencia personal o 
contextual, convirtiéndose muchas veces en clases monótonas, carentes de significado y repetitivas; además, se determinó que los contenidos curriculares no estaban planteados desde una perspectiva estructurada en relación con los niveles de complejidad de los procesos de pensamiento. Esto y según García (2015, p.19) puede tener su justificación en aspectos motivacionales de algunos maestros, quienes al ver las diversas limitantes del entorno como lo son la precaria infraestructura de las instituciones oficiales del país, especialmente aquellas de zonas rurales como la IE EI Siglo, Ios insuficientes recursos de tipo didáctico, virtual y de bienestar y, la falta de apoyo administrativo y gubernamental para la capacitación docente y el mejoramiento de sus condiciones profesionales y socioeconómicas, se limitan a ser transmisores de contenido dejando de lado la innovación mediante estrategias que dinamicen su quehacer y aunque no es excusa, si es un factor que influye grandemente.

Por su parte, los resultados obtenidos concuerdan con los reportados por Daza y Moreno (2010, p.24) y por García (2015, p.34), quienes señalaron que los docentes propician el diálogo y la participación de los estudiantes, aunque no es percibido de esta manera por los estudiantes y, aunque los docentes realicen un acompañamiento en clases, no es así desde el hogar, esto en conjunto con el bajo nivel de escolaridad de los padres de familia y su condición socioeconómica, deriva en la falta de una cultura de estudio que percibe el docente. En contraposición, la encuesta reflejó que tanto los alumnos como los docentes consideran que las clases se desarrollan en un ambiente cordial y agradable, que propicia la interacción; por tanto, existen evidencias que en el proceso de enseñanza aprendizaje de las ciencias naturales en la Institución Educativa El Siglo del municipio de Ciénaga de Oro hay prevalencia de métodos tradicionales, con algunas transiciones hacia lo constructivista, faltando claridad en su estructuración e inclusión en el desarrollo de las clases, en las estrategias de evaluación que se hacen de forma memorística y superficial y, en los recursos empleados, ya que estos fueron de naturaleza enciclopedista y correspondieron al modelo de transmisión y recepción de conocimientos (Albán, 2010, p.46).

Acorde a los resultados de la encuesta se puede mencionar que en la IE El Siglo se empleaban ocasionalmente actividades que incluían el uso de videos, el trabajo en campo o en laboratorio, actividades propias del trabajo en ciencias; los resultados reflejaron que el trabajo se centraba en actividades poco efectivas para el desarrollo de las competencias científicas tendientes a la resolución de problemas del entorno, a comprender fenómenos naturales o a familiarizarse con las formas de proceder en ciencias, indicando carencias en la contextualización adecuada del proceso de enseñanza. Finalmente, y como se mencionó anteriormente fue poco el uso de las herramientas tecnológicas, debido probablemente, a la escasa disponibilidad de materiales o equipos, o inclusive, por la falta de formación necesaria para poder aprovecharlas en el contexto educativo, dando prevalencia al uso de recursos y prácticas de corte tradicionalista (Albán, 2010, p.32; Daza y Moreno, 2010, p.23).

Por otro lado, la aplicación de la estrategia de entornos de aprendizaje autoorganizados (SOLE) permitió dejar en evidencia el efecto significativo y positivo que tienen este tipo de metodologías innovadoras en la adquisición de competencias de las ciencias naturales en los estudiantes y en la dinamización del proceso de enseñanza de los maestros. Estas estrategias pedagogías emergentes contienen enfoques que han sido modificados y adaptados a las exigencias actuales de aprendizaje, integran las tecnologías y permiten incorporar cada vez 
más elementos multidimensionales que vinculan lo social a las prácticas de construcción de conocimiento (Camacho, 2018, p.10).

De acuerdo con Doncel (2016, p.28), la metodología SOLE contribuye a que los estudiantes regulen su aprendizaje, a que sean individuos conscientes del uso de la autoorganización para el éxito académico, a que sigan metas, que hagan uso de estrategias de aprendizaje y que puedan llegar a generar cambios significativos en su entorno. De acuerdo a lo anterior, el proceso realizado en la IE EI Siglo deja en los estudiantes estrategias propias del aprendizaje autoorganizado, pues se logró que trabajaran en busca de una meta y que hicieran uso del trabajo colaborativo en las actividades, sin intervención docente, más si con el apoyo del capitán quien era un estudiante diferente en cada encuentro.

Al inicio de la implementación de la estrategia SOLE fue evidente que algunos niños y niñas no comprendían la metodología, mostrándose escépticos a participar y expresando en principio que era mejor dar las clases como habitualmente lo hacían; inicialmente se demoraban mucho y se les dificultaba buscar información y concretar sus hallazgos; sin embargo, luego de varias sesiones se fueron apropiando de la metodología, expresaron sentirse felices trabajando de esa forma y generaban sus propios interrogantes con el fin de darles solución. Teniendo en cuenta esto, se puede afirmar entonces, que la mayoría de los estudiantes aprovecharon el tiempo y supieron manejar el espacio que tenían para las actividades, así como también es importante resaltar el óptimo desarrollo de la capacidad investigativa en la consulta de contenidos que lograron crear en cada encuentro, sin intervención docente.

Mitra y Crawley (2014, p.16), considera que el aprendizaje no debe ser necesariamente intervenido por un adulto, ya que la educación es un sistema que se autoorganiza, requiriendo en su lugar un agente incentivo que despierte el interés en los estudiantes. Así que, en las sesiones de trabajo con el grupo experimental, estos fueron capaces de autoorganizarse haciendo un trabajo colaborativo y demostrando su capacidad de generar espacios compartidos para el beneficio común.

De acuerdo con Adell y Castañeda (2012, p.65), la metodología SOLE implementada en el grupo experimental del grado octavo de la IE El Siglo rompe con el esquema educativo tradicional que diseña contenidos temáticos que no se integran a las situaciones de los educandos, aunque el problema no es el contenido sino el desarrollo del mismo porque la pedagogía tradicional no conecta las temáticas a ejes multidimensionales situados en el educando, es por ello que comúnmente se escucha la frase "para que estudio eso si no me sirve para la vida" para lo que la pedagogía emergente como praxis transformativa en SOLE guía una visión de aprendizaje que recae en habilidades para la vida pues educar es también ofrecer oportunidades para que tengan lugar cambios significativos en la manera de entender y actuar en el mundo. Los estudiantes expresaron que mediante la estrategia SOLE podían aprender cosas nuevas, compartir con los compañeros y compartir sus puntos de vista con sus pares. Por tanto, la autoorganización en grupos para aprender colaborativamente fue agradable a los educandos y al compartir conjuntamente la experiencia fue significativa.

Finalmente, en cuanto a las competencias básicas de ciencias naturales se pudo notar que los niños y niñas participantes de la investigación y pertenecientes al grupo experimental 
en comparación con el grupo control se ubicaron en un nivel satisfactorio. Al finalizar la intervención con la metodología SOLE los estudiantes del grupo experimental y de acuerdo a ICFES (2018, p.6) fueron capaces de comprender el funcionamiento de los seres vivos en términos de su estructura y procesos, reconocer prácticas para el cuidado de la salud personal y de la comunidad, establecer relaciones entre materiales de diferente densidad, masa y volumen, reconocer el comportamiento de materiales cuando se someten a cambios de temperatura, reconocer que las enfermedades pueden ser de origen genético o infeccioso, hacer clasificaciones de organismos a partir de características comunes empleando el lenguaje propio de las ciencias, explicar el funcionamiento de un circuito eléctrico y la interacción de sus componentes a partir de modelos, elaborar explicaciones para ciertos fenómenos cotidianos empleando el lenguaje propio de las ciencias, elaborar conclusiones y predicciones a partir de información derivada de investigaciones científicas, reconocer patrones y regularidades en los datos derivados de una investigación científica, representar datos e información de diferentes contextos en tablas de datos, gráficas o figuras e interpretar y relacionar información presentada en tablas y distintos tipos de gráficas con conceptos de las ciencias.

\section{Conclusión}

En cuanto a las estrategias pedagógicas implementadas por los docentes en las clases de ciencias naturales se pudo establecer que las consultas en casa, los talleres con preguntas directas, las salidas al tablero y los trabajos escritos fueron las más usadas, dejando de lado actividades que involucraban la participación e interacción con los estudiantes tales como los trabajos grupales, las prácticas de laboratorio, el aprendizaje basado en problemas, las mediaciones tecnológicas y las dinámicas o los juegos. Con la investigación se determinó que los recursos didácticos y las estrategias de evaluación del aprendizaje implementadas por los docentes de ciencias naturales de la Institución Educativa El Siglo eran todas de corte tradicional y donde se premiaba la memorización de conceptos, el uso de esquemas previamente establecidos que imposibilitaban profundizar en los conocimientos adquiridos por los estudiantes y donde no se daba una significancia a lo aprendido de forma que fuera aplicable a situaciones del entorno.

La implementación de la prueba tipo Saber permitió establecer que los grupos evaluados se encontraron en condiciones similares al inicio de la investigación, no existiendo diferencias estadísticamente significativas entre ellos. Fue evidente la desmotivación y poco interés de los estudiantes al resolver el test inicial. El grupo control inició y se mantuvo en un nivel de desempeño insuficiente, con un puntaje general de 38,2 en el pretest y 36,3 en el postest, mientras que el grupo experimental se situó en un nivel mínimo al inicio de la investigación y finalizó en un nivel satisfactorio, en el pretest tuvo una calificación general de 41,9 y de 58,4 en el postest. Los resultados del grupo experimental son evidencia que existió un progreso significativo en los estudiantes al implementar la estrategia SOLE. En cuanto a las competencias básicas de ciencias naturales se pudo establecer que en la competencia uso comprensivo del conocimiento fue donde hubo la mayor dificultad y en las competencias indagación y explicación los estudiantes tuvieron un mejor desempeño. Estadísticamente en 
ninguna de las competencias existieron diferencias significativas en cuanto a la comparación de los grupos control y experimental en el pretest.

La implementación de la metodología de entornos de aprendizaje autoorganizados (SOLE), tuvo una influencia positiva y significativa en el desarrollo de las competencias básicas de los estudiantes de la Institución Educativa El Siglo del municipio de Ciénaga de Oro, notándose que con su puesta en práctica estos se mostraron participativos, seguros, motivados por aprender, trabajaron en equipo, fueron gestores de su propio aprendizaje, debatieron y compartieron lo aprendido en las clases con sus compañeros. En contraste con lo anterior, fue evidente que, para la presente investigación, las clases planeadas de forma tradicional no contribuyeron de forma significativa al aprendizaje de las ciencias naturales, en muchas ocasiones se observó el desinterés por aprender de los estudiantes, estaban distraídos y realizaban las actividades de la clase de forma mecánica sin hallarle una significancia a lo dado por el docente.

Finalmente se puede mencionar que, para que la estrategia SOLE genere cambios significativos en los estudiantes es necesario que el docente se convierta en un guía del proceso, permitiendo que los estudiantes actúen con libertad e independencia manteniendo en todo momento el orden de la clase e introducir la metodología paulatinamente, permitiendo que, tanto estudiantes como docentes se vayan acostumbrando a la nueva forma de trabajar en clases y a sus nuevos roles.

\section{Referencias}

Adell, J. y Castañeda, L. (2012). Tecnologías emergentes, ¿pedagogías emergentes? En Hernández, J., Pennesi, M, Sobrino, D. y Vázquez, A. (coord.). Tendencias emergentes con educación con TIC. Barcelona: Asociación Espiral, Educación y Tecnología. págs. 13-32. ISBN: 978-84-616-0448-7.

Albán, S. (2010). Metodologías didácticas aplicadas por los docentes en las ciencias naturales para el desarrollo de destrezas básicas. Universidad Tecnológica Equinoccial. Maestría en Educación y Desarrollo Social. Ibarra. Ecuador.

Arteaga, E., Armada, L., y Del Sol, J. (2016). La enseñanza de las ciencias en el nuevo milenio. Retos y sugerencias. Revista Universidad y Sociedad. 8 (1). pp.169-176. ISSN: 2218-3620.

Camacho, J. (2018). Prácticas pedagógicas emergentes que transforman los procesos de Enseñanza/ Aprendizaje en los niños y niñas (participantes) del proyecto SOLE Colombia en la Fundación Tiempo de Juego en Soacha (2017-2 -2018-1). Universidad Santo Tomás. Facultad de sociología. Colombia.

Cousinet, R. (2014). Qué es enseñar. Archivos de Ciencias de la Educación. 8 (8): 1-5. En Memoria Académica. Disponible en: http://www.memoria.fahce.unlp.edu.ar/art_revistas/pr.6598/pr.6598.pdf

Daza, E. y Moreno, J. (2010). El pensamiento del profesor de ciencias en ejercicio. Concepciones sobre la enseñanza y el aprendizaje de las ciencias naturales. Revista Electrónica de Enseñanza de las Ciencias Vol. 9 N³, 549-568.

Revista Assensus | Vol. 5 | Núm. 8 | 2020 |

https://doi.org/10.21897/assensus.1900 
Doncel, E. (2016). Estrategias TIC y metodología SOLE para fortalecer el inglés del proyecto "We Learn" con estudiantes de $5^{\circ}$ de primaria de una I.E.D. de Bogotá. Universidad de la Sabana. Centro de Tecnologías para la Academia. Maestría en proyectos educativos mediados por TIC. Colombia.

Fernández, M., Tuset, A., Pérez, R. y Leyva, A. (2009). Concepciones de los maestros sobre la enseñanza y el aprendizaje y sus prácticas educativas en clases de ciencias naturales. Enseñanza de las ciencias. 27(2), 287-298.

García, S. (2015). Metodologías didácticas para la enseñanza y aprendizaje de las ciencias naturales en zonas rurales del municipio de Obando - Valle del Cauca. Tesis. Universidad Nacional de Colombia. Facultad de Ingeniería y Administración. Palmira. Colombia.

González, I. (2007). La enseñanza de las ciencias naturales en la educación primaria. Tesis. Licenciatura en Educación. Universidad Pedagógica Nacional. Unidad UPN 098 DF. Oriente. México.

ICFES. (2018). Guía de Interpretación y Uso de Resultados de las pruebas Saber $3^{\circ}, 5^{\circ}$ y $9^{\circ}$. Instituto Colombiano para la Evaluación de la Educación. Bogotá. Colombia.

Lopata, M. y Schittner, V. (2014). Una experiencia local de Entornos de Aprendizaje Autoorganizados. Universidad de Buenos Aires. Congreso Iberoamericano de Ciencia, Tecnología, Innovación y Educación. ISBN: 97884-7666-210-6 - Artículo 1417.

Mitra, S. y Crawley, E. (2014). Effectiveness of Self-Organised Learning by Children: Gateshead Experiments. Journal of Education and Human Development. Vol. 3(3). ISSN: 2334-2978. Published by American Research Institute for Policy Developmen

Murillo, J., Hernández, R. y Martínez, C. (2016). ¿Qué ocurre en las aulas donde los niños y niñas no aprenden? Estudio cualitativo de aulas ineficaces en Iberoamérica. Perfiles Educativos. vol. XXXVIII, núm. 151. IISUE-UNAM.

Ortega, C. (2014). SOLE, un modelo educativo inspirado en el auto-aprendizaje. En: http://www.youngmarketing.co/sole-un-modelo-de-educacion-inspirado-en-el-auto-aprendizaje/ (Consultado: 15 de agosto de 2019).

Pita, S. y Pértegas, S. (2002). Investigación cuantitativa y cualitativa. Unidad de Epidemiología Clínica y Bioestadística. Complexo Hospitalario-Universitario Juan Canalejo. A Coruña (España). Cad Aten Primaria. 9:76-78.

Sarmiento, M. (2004). La enseñanza de las matemáticas y las nuevas tecnologías de la información y comunicación. Universitat Rovira I Virgili. ISBN: 978-84-690-8294-2 / D.L: T.1625-2007.

Zapata, M. (2015). Teorías y modelos sobre el aprendizaje en entornos conectados y ubicuos. Bases para un nuevo modelo teórico a partir de una visión crítica del "conectivismo". Education in the Knowledge Society. 16(1) 69-102. E-ISSN: 2444-8729. Universidad de Salamanca. España. 